\title{
Enculturating Conversational Agents Based on a Comparative Corpus Study
}

\author{
Afia Akhter Lipi ${ }^{1}$, Yuji Yamaoka ${ }^{1}$, Matthias Rehm², \\ and Yukiko I. Nakano ${ }^{3}$ \\ ${ }^{1}$ Dept. of Computer and Information Sciences, Tokyo University of Agriculture and \\ Technology, Japan \\ $\{50007646211,50007646208\}$ ast.tuat.ac.jp \\ ${ }^{2}$ Institute of Computer Science, Augsburg University, Germany \\ rehmainformatik.uni-augsburg.de \\ ${ }^{3}$ Dept. of Computer and Information Science, Seikei University, Japan \\ y.nakano@st.seikei.ac.jp
}

\section{Introduction}

When encountering people who have a different cultural background from our own, many of us feel uncomfortable because gestures and facial expressions may not be familiar to us. Thus, to enhance the believability of conversational agents, culture-specific nonverbal behaviors should be implemented into the agents. In our previous study [1], with the goal of building a user interface that incorporates a user's cultural background, we have collected comparative conversation corpus in Germany and Japan, and investigated the differences in gestures and posture shifts between these two countries. Based on [1], this paper reports a more detailed analysis about posture shifts, and proposes a chat system with an embodied conversational agent (ECA) that can act as a language trainer.

\section{Analysis of Comparative Corpus}

For the empirical basis of our ECA, we calculated statistics for speakers' posture shift frequency and distribution in our comparative corpus. The results are shown in Table 1. We use Bull's posture coding scheme in [2], but change the abbreviations for better readability. Each speaker-turn is divided into three sections: first $(\mathrm{F})$, middle $(\mathrm{M})$, and end (E). The "Ratio" column shows the distribution of posture shift occurrences.

For example, head postures most frequently occurred at (F) in both the Japanese and German data (36\% in the German data and $31 \%$ in the Japanese data). Leg and Head postures were not very different in each culture. Both German and Japanese rested on the right or left leg throughout the conversation, and the most frequent head posture was "Turn head away". This head posture was reported as a typical turn-taking signal in Duncan's analysis of American people [3]. Significant difference was found in arm postures. German people frequently put their hands in their pockets or folded their arms. On the contrary, Japanese people put one hand on their face at the beginning of a turn, and joined their hands or put one hand on top of the other with fingers touching the 
wrist at the middle and end of a turn. These results are useful in deciding when the agent should change her/his posture, and what types of posture are appropriate according to a given culture.

Table 1. Frequently occurring postures in each section of a turn

\begin{tabular}{|c|c|c|l|l||c|l|l|}
\hline & & \multicolumn{3}{|c||}{ Germany } & \multicolumn{3}{|c|}{ Japan } \\
\cline { 2 - 8 } & Section & Ratio & \multicolumn{1}{|c|}{ Frequency \# 1 } & \multicolumn{1}{|c|}{ Frequency \# 2 } & Ratio & \multicolumn{1}{|c|}{ Frequency \# 1 } & \multicolumn{1}{|c|}{ Frequency \# 2 } \\
\hline \multirow{4}{*}{ Leg } & F & $39 \%$ & Weight on left leg (33\%) & Weight on right leg (24\%) & $28 \%$ & Weight on right leg (45\%) & Weight on left leg (25\%) \\
& M & $22 \%$ & Weight on right leg (42\%) & Weight on left leg (42\%) & $26 \%$ & Weight on right leg (43\%) & Stop leaning sideways (21\%) \\
& E & $34 \%$ & Weight on left leg (38\%) & Weight on right leg (25\%) & $31 \%$ & Weight on right leg (37\%) & Stop leaning sideways (26\%) \\
\hline \multirow{3}{*}{ Head } & F & $36 \%$ & Turn head away (40\%) & Straighten head (32\%) & $31 \%$ & Straighten head (39\%) & Turn head away (29\%) \\
& M & $29 \%$ & Straighten head (31\%) & Turn head away (25\%) & $23 \%$ & Straighten head (42\%) & Turn head away (24\%) \\
& E & $28 \%$ & Straighten head (43\%) & Turn head away (20\%) & $25 \%$ & Turn head away (50\%) & Straighten head (32\%) \\
\hline \multirow{4}{*}{ Arm } & F & $33 \%$ & Put hands in pockets (56\%) & Put hand on elbow (13\%) & $27 \%$ & Puts hand on face (30\%) & JoinHands (24\%) \\
& M & $30 \%$ & Put hands in pockets (57\%) & Fold arms (14\%) & $23 \%$ & Puts hand on face (28\%) & JoinHands (28\%) \\
& E & $32 \%$ & Put hands in pockets (34\%) & Fold arms (25\%) & $32 \%$ & JoinHands (35\%) & Puts hand on wrist (19\%) \\
\hline
\end{tabular}

\section{Enculturated Language-Trainer Agents}

Based on our corpus study, we came up with the idea of implementing such culture-specific behaviors in ECAs. In Fig. 1 a student first chooses which language s/he wants to learn. When s/he chooses Japanese, a human Japanese teacher types in Japanese text. The text is sent to TTS and appropriate postures

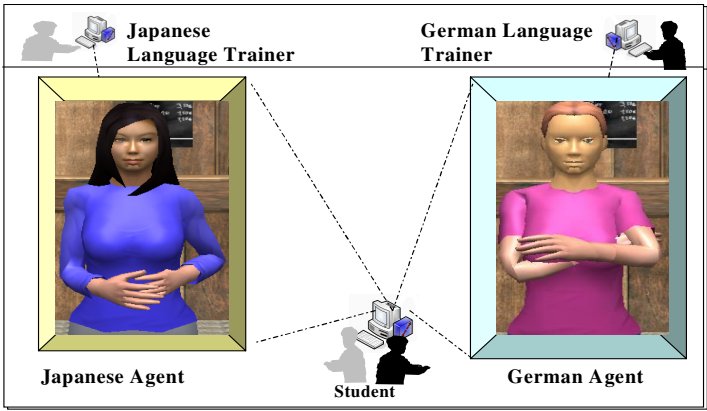

Fig. 1. Language- trainer Agents are determined based on the empirical data in Table 1. Finally, the speech sound and agent-posture animations are produced on the student's computer display. Likewise, if the student chooses German, then German text typed by a real German teacher is converted into speech accompanied by typical German posture animations. Thus, the system not only teaches language, but also makes the learner familiar with the culture-specific non-verbal behaviors. In the future, we hope that this system can be used as a distance-learning system by which a user can train her/himself how to smoothly communicate with people from other cultures.

Acknowledgment. This work is funded by the German Research Foundation (DFG) under research grant RE 2619/2-1 (CUBE-G) and the Japan Society for the Promotion of Science (JSPS) under a Grant-in-Aid for Scientific Research (C) (19500104). 


\section{References}

1. Rehm, M., et al.: Creating a Standardized Corpus of Multimodal Interactions for Enculturating Conversational Interfaces. In: Proceedings of Workshop on Enculturating Conversational Interfaces by Socio-cultural Aspects of Communication, 2008 International Conference on Intelligent User Interfaces (IUI 2008) (2008)

2. Bull, P.E.: Posture and Gesture. Pergamon Press, Oxford (1987)

3. Duncan, S.: On the structure of speaker-auditor interaction during speaking turns. Language in Society 3, 161-180 (1974) 